\title{
Etude dendrométrique de Lophira lanceolata de la forêt classée de Toui-Kilibo et ses environs au Bénin
}

\author{
Roméo Worou ADJERAN ${ }^{1 *}$, Toussaint Olou LOUGBEGNON ${ }^{2}$ et \\ Gaudence Julien DJEGO ${ }^{1}$ \\ ${ }^{1}$ Laboratoire d'Ecologie Appliquée (LEA), Faculté des Sciences Agronomiques (FSA), Université \\ d'Abomey-Calavi (UAC), O1BP 526 Cotonou, République du Bénin. \\ ${ }^{2}$ Ecole de Foresterie et d'Ingénierie du Bois (EFIB), Université Nationale d'Agriculture (UNA), \\ BP: 43 Kétou, Bénin. \\ *Auteur correspondant, E-mail : adjeranromeo@gmail.com
}

\section{RESUME}

La connaissance des paramètres dendrométriques d'une espèce est nécessaire pour sa conservation durable. Elle permet d'évaluer les ressources disponibles et de lutter contre sa destruction. Cette étude analyse les paramètres dendrométriques de Lophira lanceolata de la forêt classée de Toui-Kilibo et ses environs au Bénin. Les données dendrométriques sont collectées à l'intérieur de 64 placettes carrées de 30 mètres de côté. L'analyse des données prend en compte la contribution en surface terrière de Lophira lanceolata, la densité relative, la dominance relative, la fréquence, l'amplitude d'habitat, le diamètre moyen par les types de formations végétales. Le diamètre moyen de Lophira lanceolata $(25,5 \mathrm{~cm})$, la hauteur moyenne $(15,21 \mathrm{~cm})$ et la surface terrière moyenne $\left(0,63 \mathrm{~m}^{2} / \mathrm{ha}\right)$ de la forêt claire et savane boisée sont supérieurs aux autres types de formations végétales. Ce constat est fait également sur la fréquence, la densité relative, la dominance relative. La contribution en surface terrière de Lophira lanceolata est de 9,363\% en forêt claire et savane boisée, 7,398\% en savane arborée et arbustive et $2,65 \%$ en champs et jachères. En forêt claire et savane boisée, lorsque le diamètre de Lophira lanceolata s'accroît d'un centimètre, sa surface terrière augmente de $0,043 \mathrm{~m}^{2} /$ ha. L'étude permet de connaître les ressources disponibles sur Lophira lanceolata et de prendre les décisions sur son aménagement.

(C) 2020 International Formulae Group. All rights reserved.

Mots clés : Lophira lanceolata, paramètres dendrométriques, forêt, savane, Bénin.

\section{Dendrometric study of Lophira lanceolata of the Toui-Kilibo classified forest and its surroundings in Benin}

\begin{abstract}
Knowledge of the dendrometric parameters of a species is necessary for its sustainable conservation. It makes it possible to assess the resources available and to combat its destruction. This study analyses the dendrometric parameters of Lophira lanceolata of the classified forest of Toui-Kilibo and its surroundings in Benin. Dendrometric data is collected inside 64 square plots 30 meters wide. Data analysis concerns the contribution in basal area of Lophira lanceolata, the relative density, the relative dominance, the frequency, the range of habitat, the average diameter by the types of plant formations. The average diameter of Lophira
\end{abstract}


lanceolata $(25.5 \mathrm{~cm})$, the average height $(15.21 \mathrm{~cm})$ and the average basal area $\left(0.63 \mathrm{~m}^{2} / \mathrm{ha}\right)$ of the open forest and wooded savannah are superior to other types of plant formations. This observation is also made on the frequency, the relative density, the relative dominance. The contribution in basal area of Lophira lanceolata is 9.363\% in open forest and wooded savannah, $7.398 \%$ in tree and shrubby savannah and $2.65 \%$ in fields and fallows. In open forest and wooded savannah, when the diameter of Lophira lanceolata increases by one centimeter its basal area increases by $0.043 \mathrm{~m}^{2} / \mathrm{ha}$. The study makes it possible to know the resources available on Lophira lanceolata and to take decisions on its development.

(C) 2020 International Formulae Group. All rights reserved.

Keywords: Lophira lanceolata, dendrometric parameters, forest, savanna, Benin.

\section{INTRODUCTION}

Pour garantir la durabilité des plantes, une bonne stratégie de conservation et de restauration dans les milieux naturels est importante (Fandohan et al., 2008). La réussite de cette stratégie nécessite la connaissance des paramètres dendrométriques des espèces. L'objectif de la dendrométrie est d'estimer le plus précisément possible la taille, la forme, et le volume d'arbres d'une forêt naturelle ou d'une plantation (Sagbo, 2000). Cette dendrométrie est nécessaire pour apprécier la population d'une espèce. De ce fait, la connaissance de la distribution des diamètres d'un peuplement est un grand intérêt pour son aménagement durable, ceci permettra d'évaluer les ressources disponibles et de planifier les prélèvements futurs (Fonton et Sagbo, 2004). Ainsi, les variables dendrométriques sont indispensables à la prise de décision en aménagement forestier (Fortin et al., 2009). Pour Dicko et al. (2017), la bonne maîtrise de la structure des populations d'une espèce est nécessaire pour l'efficacité des programmes d'aménagement. Les structures en diamètre et en hauteur d'une espèce végétale sont révélatrices des évènements liés à la vie des peuplements (Garba et al., 2020).

C'est dans cette optique que cette étude vise à déterminer quelques paramètres dendrométriques de Lophira lanceolata de la forêt classée de Toui-Kilibo et ses environs au Bénin en vue d'obtenir une base de données fiables pour son aménagement. Igué et al (2010) ont affirmé que les superficies des formations forestières cette forêt se sont réduites entre 1975 et 2003, jusqu'à $32 \%$ pour les forêts claires et savanes boisées, 30\% pour les forêts galeries puis $10 \%$ pour les forêts denses sèches. La recherche des données dendrométriques sur Lophira lanceolata est importante puisque l'espèce subit d'énormes pressions dans le secteur d'étude. Les populations sont à la recherche des organes de l'espèce pour satisfaire leurs besoins sociaux. Donc une base de données serait nécessaire pour l'aménagement des peuplements de Lophira lanceolata au Bénin. Cette étude analyse les paramètres dendrométriques de Lophira lanceolata de la forêt classée de TouiKilibo et ses environs au Bénin. Elle permet de prendre les décisions sur l'aménagement des peuplements de cette espèce. Elle permet également de ressortir les difficultés de sa conservation en vue de prendre des mesures appropriées pour sa conservation durable.

\section{MATERIEL ET METHODES}

\section{Méthode de collecte des données}

Le secteur d'étude est situé dans la commune de Ouèssè, département des Collines au Bénin. La forêt classée de Toui-Kilibo appartient à un climat Soudano-guinéen (Djogbénou et al., 2008).

Pour la collecte des données, deux catégories de placeaux sont installées. La première catégorie concerne les grands placeaux carrés de dimensions 500 mètres de côtés (soit $250000 \mathrm{~m}^{2}$ ). La deuxième catégorie fait allusion aux placettes carrées de dimension 30 mètres de côtés (soit $900 \mathrm{~m}^{2}$ ). Sur chaque grand placeau de $250000 \mathrm{~m}^{2}, 8$ placettes sont installées. Les 8 placettes de chaque grand placeau suivent 8 directions à savoir : Nord $(\mathrm{N})$ à 0 , Nord Est (NE) à 45, Est (E) à $90^{\circ}$, Sud Est (SE) à 135 , Sud (S) à $180^{\circ}$, Sud-Ouest (SW) à 
$225^{\circ}$, Ouest (W) à $270^{\circ}$, Nord-Ouest (NW) à $315^{\circ}$. La Figure 1 présente la situation géographique du secteur d'étude avec les 8 grands placeaux. Alors que la Figure 2 montre l'orientation de 8 placettes dans un grand placeau.

A l'intérieur de chaque placette installée, le nombre d'espèces est dénombré. De même la hauteur et le diamètre à hauteur de poitrine de tous les arbres sont mesurés.

\section{Traitement et analyse des données}

Plusieurs paramètres dendrométriques sont utilisés pour l'analyse des données. Il s'agit de :

\section{La densité du peuplement $(N)$}

C'est le nombre moyen d'arbres sur pied ramené à l'hectare.

$\mathbf{N}=\frac{\boldsymbol{n}}{\boldsymbol{s}}$

Avec $\mathbf{n}$ le nombre d'arbres d'une placette et $\mathbf{s}$ est la superficie de la placette en ha.

\section{La surface terrière $(G)$ en $\mathrm{m}^{2} / \mathrm{ha}$}

C'est la somme des sections transversales à 1,30 mètre de hauteur au-dessus du sol des arbres de la placette.

$$
\mathbf{G}=\frac{\pi}{4 \mathbf{s}} \sum_{\mathbf{i}=1}^{\mathbf{n}} d_{i}{ }^{2}
$$

$\mathbf{d}_{\mathbf{i}}$ étant le diamètre de l'arbre et $\mathbf{S}$ est la superficie de la placette

Le diamètre des arbres de surface terrière $(D)$

Elle est utilisée pour les arbres à fourchues avant 1,3 m du sol. Les différentes fourchues sont mesurées et les calculs du diamètre quadratique sont établis (Thiombiano et al., 2016). Sa formule est :

$\mathrm{D}=\sqrt{\frac{1}{\mathrm{n}} \sum_{\mathrm{i}=1}^{\mathrm{n}} \mathrm{di}^{2}}$

Avec $\mathbf{n}$ le nombre de fourchues et $\mathbf{d}_{\mathbf{i}}$ le diamètre de chaque fourchues.

\section{La hauteur moyenne de Lorey $(\mathrm{H})$}

C'est une hauteur moyenne des arbres pondérée par surface terrière (Glèlè Kakaï, 2008).

$H=\frac{\sum_{i=1}^{n} g_{i} h_{i}}{\sum_{i=1}^{n} g_{i}}$ avec $g_{i}=\frac{\pi}{4 s} \sum_{i=1}^{n} d i^{2}$

$\mathbf{g}_{\mathbf{i}}$ étant la surface terrière (en $\left.\mathrm{m}^{2} / \mathrm{ha}\right)$, hi la hauteur de l'arbre i.
Contribution en surface terrière (Cs) en \%

C'est la part de Lophira lanceolata dans la surface terrière au niveau des arbres de la placette.

Cs $=\frac{\mathbf{G p}}{\mathbf{G}} \times 100$

Gp étant la surface terrière des arbres de Lophira lanceolata, G la surface terrière de tous les arbres de la placette.

\section{L'Indice de Blackman ( $\left.I_{B}\right)$}

Cet indice permet de caractériser la distribution spatiale des populations de Lophira lanceolata par formation végétale (Glèlè Kakaï, 2008).

$\mathrm{I}_{\mathrm{B}}=\boldsymbol{\sigma}^{2} N \frac{\mathbf{1}}{\mu \mathrm{N}}$

$\boldsymbol{\sigma}^{2} \mathbf{N}$ étant la variance de la densité, $\boldsymbol{\mu}_{\mathbf{N}}$ la moyenne de la densité.

Densité relative d'une espèce (Densr) en \%

Elle est trouvée en faisant le rapport du nombre d'individus de l'espèce au nombre total d'individus (Thiombiano et al., 2016).

Densr

$$
=\frac{\text { Nombre d'individusde l'espèce }}{\text { Nombre total d'individus }} \times 100
$$

\section{Dominance relative d'une espèce (Domr) en} $\%$

Elle est trouvée par le rapport de la surface terrière totale d'une espèce et de la surface terrière totale de toutes les espèces (Thiombiano et al., 2016).

Domr

$=\frac{\text { Surface terrière totale } \mathrm{d}^{\prime} \text { une espèce }}{\text { Surface terrière totale de toutes les espèces }} \times 100$

\section{Fréquence d'une espèce}

Fréquence d'une espèce représente le nombre de relevés dans lequel l'espèce est présente sur le nombre total de relevés (Thiombiano et al., 2016).

Fréquence

$=\frac{\text { Nombre de relevés dans lequel 'espèce est présente }}{\text { Nombre total de relevés }}$

\section{Amplitude d'habitat (AH)}

Mesure l'hétérogénéité d'une espèce le long d'une séquence d'habitat. Plus la valeur de l'amplitude est grande, plus l'espèce est souple dans ces normes de réaction ou descripteur considéré. Plus elle est faible, plus l'espèce est exigeante. AH vaut 1 quand l'espèce n'est présente que dans un milieu et $\mathbf{n}$ 
quand l'espèce est répandue de manière égale dans les $\mathbf{n}$ milieux.

$$
A H=e^{H} \quad \text { avec } H=\sum p_{i} \log 2 p_{i}
$$

Relation entre hauteur (H)-diamètre (D) surface terrière $(\mathbf{G})$

La relation entre la hauteur $(\mathrm{H})$, le diamètre (D) et la surface terrière est testée part la formule suivante :

\section{$\mathbf{G}=\mathbf{a}+\mathbf{b D}+\mathbf{c H}$}

$\mathrm{G}$ étant la surface terrière en $\mathrm{m}^{2} / \mathrm{ha}$, $\mathrm{D}$ le diamètre en $\mathrm{cm}$ et $\mathrm{H}$ la hauteur en mètre. Le logiciel SPSS 16.0 est utilisé à cet effet.

\section{Relations entre hauteur $(\mathrm{H})$-diamètre $(\mathrm{D})$ et entre la surface terrière $(G)$-diamètre $(D)$}

Les relations entre la hauteur $(\mathrm{H})$ et le diamètre (D) d'une part et la surface terrière (G) et le diamètre (D) d'autre part de Lophira lanceolata sont appréciées par cinq modèles de régression linéaire. Les équations suivantes traduisent ces modèles.
- Modèle de Trorey

$$
\begin{aligned}
& \mathrm{H}=\mathrm{a}-\mathrm{bD}+2 \mathrm{CD}^{2} \\
& \mathrm{G}=\mathrm{a}-\mathrm{bD}+2 \mathrm{CD}^{2}
\end{aligned}
$$

- Modèle de Hendricksen

$$
\begin{aligned}
& \mathrm{H}=\mathrm{a}+\mathrm{bLnD} \\
& \mathrm{G}=\mathrm{a}+\mathrm{bLnD}
\end{aligned}
$$

\section{- Modèle de Stoffels et de Van Soest}

$$
\mathrm{LnH}=\mathrm{a}+\mathrm{bLnD}
$$$$
\mathrm{LnG}=\mathrm{a}+\mathrm{bLnD}
$$

\section{- Modèles de Avery et Burkart}

$$
\begin{aligned}
\mathrm{LnH} & =\mathrm{a}-\mathrm{b} \frac{1}{D} \\
\mathrm{LnG} & =\mathrm{a}-\mathrm{b} \frac{1}{D}
\end{aligned}
$$

Les constantes $\mathrm{a}$, b et $\mathrm{c}$ sont les coefficients de régression. Ces modèles de régression permettent d'apprécier le lien qui existe entre la hauteur $(\mathrm{H})$ et le diamètre $(\mathrm{D})$ d'une part, et entre la surface terrière $(G)$ et le diamètre (D) d'autre part de Lophira lanceolata.

\section{- Régression linéaire simple}

$$
\begin{aligned}
& \mathrm{H}=\mathrm{a}+\mathrm{bD} \\
& \mathrm{G}=\mathrm{a}+\mathrm{bD}
\end{aligned}
$$

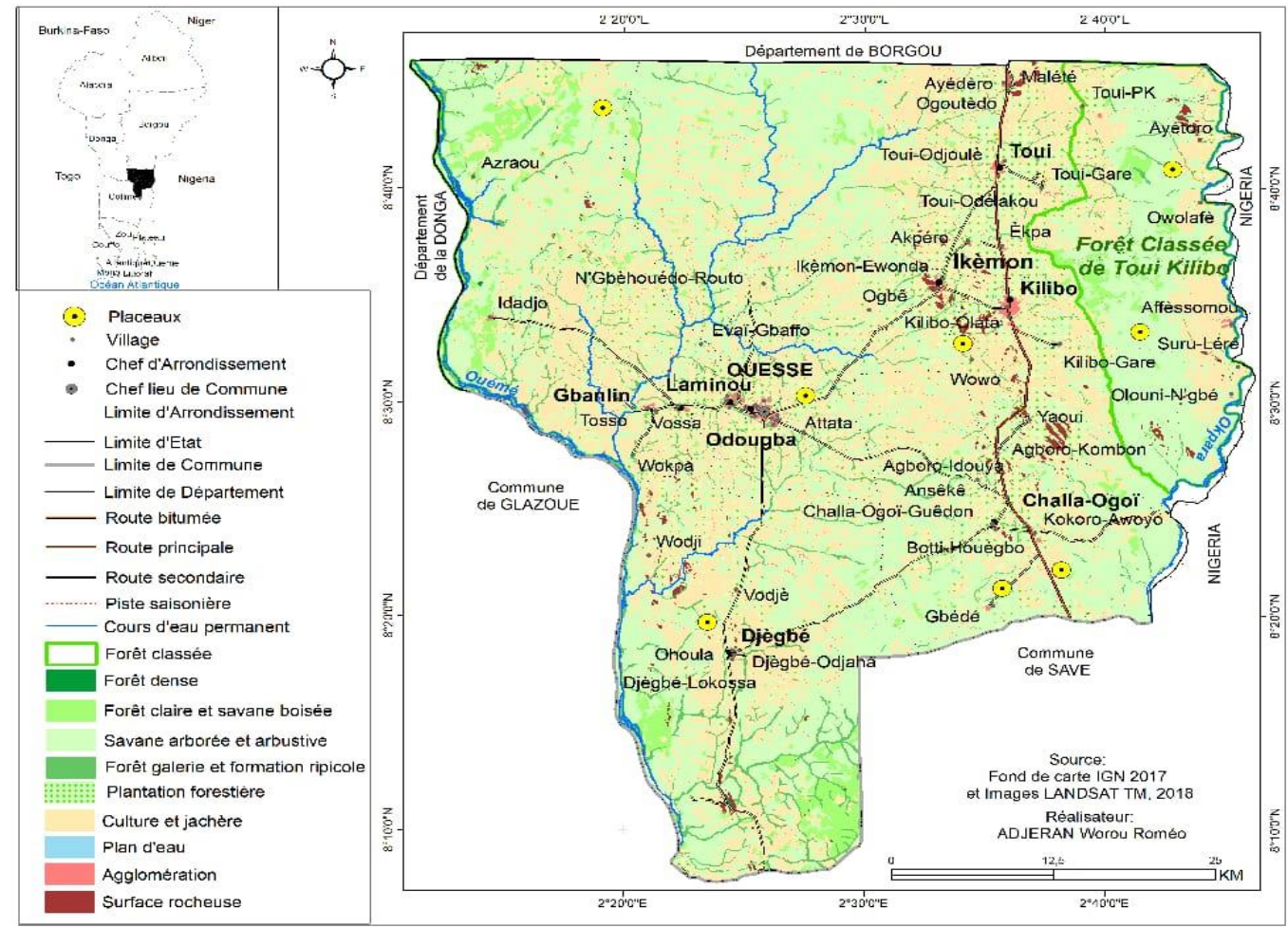

Figure 1 : Localisation des points centraux des randomisées de $500 \mathrm{~m}$ x $500 \mathrm{~m}$ sur une carte. 


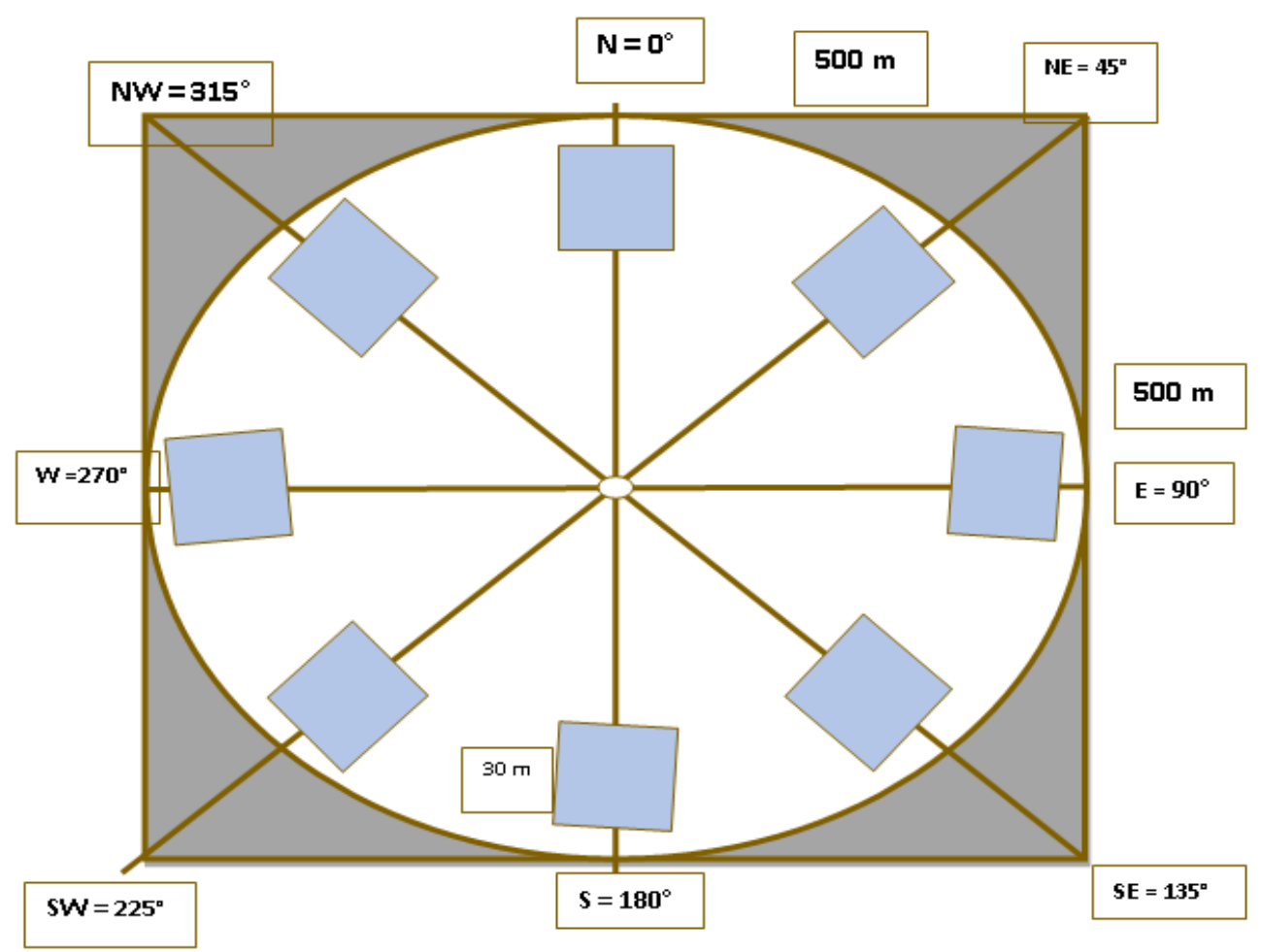

Figure 2: Schéma d'installation de 8 placettes au sein d'un grand placeau de $250000 \mathrm{~m}$.

\section{RESULTATS}

Comparaison de diamètre, hauteur, surface terrière, de peuplement global et des individus de Lophira lanceolata

Le Tableau 1 présente la statistique descriptive des paramètres dendrométriques des peuplements de Lophira lanceolata en forêt claire et en savane boisée (FCSB), en savane arborée et arbustive ( $\mathrm{SAB})$ et en champs et jachères $(\mathrm{CJ})$ de la région de TouiKilibo et ses environs. Au niveau de la forêt claire et savane boisée, la moyenne des paramètres dendrométriques des arbres de toutes les espèces confondues est de $22,17 \mathrm{~cm}$, $10,35 \mathrm{~m}$ et $0,52 \mathrm{~m}^{2} /$ ha respectivement pour le diamètre moyen, la hauteur moyenne et la surface terrière moyenne. Dans les savanes arborées et arbustives, le diamètre moyen des arbres est de 18,06 $\mathrm{cm}$, avec une hauteur moyenne de $8,27 \mathrm{~m}$ et une surface terrière moyenne de $0,36 \mathrm{~m}^{2} /$ ha. Pour les champs et jachères, la valeur du diamètre moyen des arbres est de $21,48 \mathrm{~cm}$, avec une hauteur moyenne de $8,20 \mathrm{~m}$ et une surface terrière moyenne de $0,50 \mathrm{~m}^{2} / \mathrm{ha}$. Alors que pour les individus de Lophira lanceolata, les paramètres dendrométriques se présentent comme suit : le diamètre moyen est de 25,50 $\mathrm{cm}, 18,13 \mathrm{~cm}$ et $19,84 \mathrm{~cm}$ respectivement pour la forêt claire et savane boisée, la savane arborée et arbustive puis pour les champs et jachères. La hauteur moyenne est de $15,21 \mathrm{~m}$, $9,08 \mathrm{~m}$ et $8,12 \mathrm{~m}$ respectivement pour la forêt claire et savane boisée, la savane arborée et arbustive puis pour les champs et jachères. La surface terrière moyenne est de $0,63 \mathrm{~m}^{2} / \mathrm{ha}$, $0,43 \mathrm{~m}^{2} /$ ha et $0,41 \mathrm{~m}^{2} /$ ha respectivement pour la forêt claire et savane boisée, la savane arborée et arbustive puis les champs et jachères. Le diamètre maximum $(36 \mathrm{~cm})$, la hauteur maximum $(26,50 \mathrm{~m})$ ainsi que la surface terrière maximum $\left(1,13 \mathrm{~m}^{2} / \mathrm{ha}\right)$ sont trouvés à l'intérieur de la forêt et non en périphérie. Donc les paramètres dendrométriques de Lophira lanceolata de l'intérieur de la forêt ont des valeurs plus élevées que ceux des zones périphériques. Ceci parce que la forêt est plus protégée que les 
zones environnantes. Les effets de la déforestation et de l'exploitation de Lophira lanceolata ne sont pas les mêmes entre l'intérieur de la forêt et ses zones environnantes. $\mathrm{La}$ déforestation et la surexploitation de l'espèce sont plus prononcées aux environs de la forêt qu'à l'intérieur. C'est ce qui justifie la présence des pieds de Lophira lanceolata à forts diamètres et hauteurs à l'intérieur de la forêt.

\section{Surface terrière totale de toutes les espèces confondues et des arbres de Lophira lanceolata au niveau de la forêt claire et savane boisée de Toui-Kilibo}

La Figure 3 présente la surface terrière totale de toutes les espèces confondues et des arbres de Lophira lanceolata à l'intérieur de la forêt claire et savane boisée de Toui-Kilibo. Cette surface terrière est dispersée suivant les placettes installées.

La boîte de dispersion de la surface terrière totale par placette de toutes les espèces confondues à l'intérieur de la forêt visualise une forte surface terrière de la placette 46 avec $21,45 \mathrm{~m}^{2} / \mathrm{ha}$, et une faible surface terrière de la placette 30 avec $7,39 \mathrm{~m}^{2} / \mathrm{h}$. Cette observation de la surface terrière sur les pieds de Lophira lanceolata donne une surface terrière de 1,91 $\mathrm{m}^{2} /$ ha sur la placette 46 et une surface terrière de $0,15 \mathrm{~m}^{2} / \mathrm{ha}$ de la placette 28 . Les fortes valeurs de la surface terrière indiquent que l'espèce est bien développée dans ces placettes.

\section{Surface terrière totale de toutes les espèces confondues au sein des placeaux au niveau des savanes arborées et arbustives}

La Figure 4 fait l'aperçu de la surface terrière totale de toutes les espèces confondues et des arbres de Lophira lanceolata au niveau de la savane arborée et arbustive. La boîte de dispersion visualise deux valeurs de surfaces terrières supérieures à $15 \mathrm{~m}^{2} /$ ha et une valeur inférieure à $1 \mathrm{~m}^{2} / \mathrm{ha}$ au niveau de toutes les espèces confondues des savanes arborées et arbustives aux environs de la forêt claire. Pour ce qui est des individus de Lophira lanceolata, la boîte de dispersion indique une valeur extrême supérieure à $14 \mathrm{~m}^{2} / \mathrm{ha}$ et une valeur minimale inférieure à $3 \mathrm{~m}^{2} / \mathrm{ha}$. Nous pouvons dire que la surface terrière de Lophira lanceolata au niveau des savanes arborées n'est pas négligeable.

Surface terrière totale de toutes les espèces confondues au sein des placeaux au niveau des champs et jachères

La Figure 5 présente la surface terrière totale de toutes les espèces confondues et des arbres de Lophira lanceolata au niveau des champs et jachères. Pour toutes les espèces confondues, la boîte de dispersion visualise deux valeurs de surface terrière supérieures à 5 $\mathrm{m}^{2} /$ ha (placettes 16) et (placette 45). S'agissant des individus de Lophira lanceolata, la valeur extrême est de $10 \mathrm{~m}^{2} /$ ha (placette 57). Cette forte valeur observée au niveau des champs est liée au fait que quelques agriculteurs conservent l'espèce dans leurs agrosystèmes pour les usages multiples. En effet, Lophira lanceolata est une espèce fortement recherchée par les guérisseurs traditionnels. Donc la conservation de cette espèce dans les champs est bénéfique et permet aux cultivateurs d'avoir l'espèce à proximité pour leurs besoins médicinaux.

\section{Contribution en surface terrière des arbres de Lophira lanceolata}

La Figure 6 renseigne sur la contribution en surface terrière des arbres de Lophira lanceolata dans le secteur d'étude. De l'analyse de la Figure 6, la contribution en surface terrière de Lophira lanceolata en forêt claire et savane boisée de Toui-Kilibo est de $9,363 \%, 7,398 \%$ en savane arborée et arbustive puis $2,65 \%$ en champs et jachères. Donc, on peut retenir que la contribution en surface terrière de Lophira lanceolata décroît de la zone protégée vers la zone non protégée.

Fréquence, densité relative, dominance relative, Amplitude d'habitat, et Indice de Blackman des arbres de Lophira lanceolata par types de formations végétales

Le Tableau 2 présente la fréquence, la densité relative, la dominance relative, l'amplitude d'habitat, et l'indice de Blackman des arbres de Lophira lanceolata suivant les formations végétales. 
La fréquence, la densité relative, et la dominance relative de l'espèce issues de la forêt claire et savane boisée ont des valeurs élevées qu'aux zones de savane arborée et arbustive et des champs et jachères. Alors la faible valeur de l'amplitude d'habitat prouve que l'espèce est exigeante.

Par ailleurs, les paramètres dendrométriques des essences de valeur des peuplements à dominance Lophira lanceolata en champs et jachères ont été présentés dans le Tableau 3. Au niveau des champs et jachères, 10 espèces compagnes avec Lophira lanceolata dont le diamètre est supérieur à 10 $\mathrm{cm}$ ont été recensées. Il s'agit de: Afzelia africana, Anogeissus leiocarpus, Combretum glutinosum, Ficus sur, Khaya senegalensis, Lannea barteri, Pterocarpus erinaceus, Tectona grandis, Vitellaria paradoxa, Vitex doniana. Parmi ces espèces, Pterocarpus erinaceus est la plus dense avec 22,22 pieds à l'hectare. L'espèce ayant la plus grande surface terrière et le plus grand diamètre est Vitellaria paradoxa avec respectivement $12,24 \mathrm{~m}^{2} /$ ha et $38,5 \mathrm{~cm}$. L'espèce ayant la plus grande hauteur est Khaya senegalensis avec 12,5 mètres.

$\mathrm{Au}$ niveau des savanes arborée et arbustives, les paramètres dendrométriques des essences de valeur des peuplements à dominance Lophira lanceolata sont renseignés dans le Tableau 4. L'analyse du Tableau 4 montre que 20 espèces compagnes Lophira lanceolata. Notons qu'Anogeissus leiocarpus est la plus dense avec 369,44 pieds/ha. Daniella oliveri et Vitellaria paradoxa viennent en deuxième et troisième position avec respectivement 275 et 191,67 arbre /ha.

Au niveau de la Forêt claire et Savane boisée, les paramètres dendrométriques des essences de valeur des peuplements à dominance Lophira lanceolata sont renseignés dans le Tableau 5. L'analyse de Tableau 5 montre que 23 espèces ligneuses compagnes avec Lophira lanceolata. Les espèces les plus denses concernent: Anogeissus leiocarpus (74,44 arbres/ha), Daniella oliveri (57,78 arbres/ha), Isoberlinia doka (46,67 arbres/ha), Burkea africana (33,33 arbres/ha), Combretum collinum (27,78 arbres/ha), Piliostigma toningii (26,67 arbres/ha), Crossopteryx febrifuga (26,67 arbres à l'hectare), Vitellaria paradoxa (25,56 arbres/ha). Toujours dans ce groupement, les espèces ayant la plus grande surface terrière concernent: Isoberlinia doka (47,86 $\left.\mathrm{m}^{2} / \mathrm{ha}\right)$, Daniella oliveri $\left(41,96 \mathrm{~m}^{2} / \mathrm{ha}\right)$, Anogeissus leiocarpus $\left(34,48 \quad \mathrm{~m}^{2} / \mathrm{ha}\right)$, Vitellaria paradoxa $\left(17,08 \mathrm{~m}^{2} / \mathrm{ha}\right)$, Burkea africana $\left(16,9 \mathrm{~m}^{2} / \mathrm{ha}\right)$. Ces dernières espèces sont des espèces de bois d'œuvre.

Relations entre la surface terrière $(G)$ diamètre (D)-hauteur (H) des arbres de Lophira lanceolata

Le Tableau 6 fait le point de l'analyse de la régression linéaire entre la surface terrière $(\mathrm{G})$, le diamètre (D) et la hauteur $(\mathrm{H})$ des arbres de Lophira lanceolata. En forêt claire et savane boisée de Toui-Kilibo, lorsque le diamètre de Lophira lanceolata s'accroît d'un centimètre, sa surface terrière augmente de $0,043 \mathrm{~m}^{2} / \mathrm{ha}$. En savane arborée et arbustive, l'accroissement d'un centimètre de Lophira lanceolata engendre un accroissement de $0,044 \mathrm{~m}^{2} /$ ha de sa surface terrière.

\section{Forme de l'arbre de Lophira lanceolata au niveau de la forêt claire et la savane boisée}

Les résultats d'ajustement des données de la hauteur (H) et du diamètre (D) de Lophira lanceolata aux différents modèles de régression permettent de noter que l'ajustement est significatif sur quatre modèles $(p<0,05)$. Ces quatre modèles sont relatifs au modèle linéaire simple $(\mathrm{p}=0,018)$, au modèle de Hendricksen ( $p=0,024)$, au modèle de Stoffels et de Van Soest $(\mathrm{p}=0,026)$, au modèle de Avery et Burkart $(0,038)$. Mais l'ajustement n'est pas significatif sur le modèle de Trorey $(\mathrm{p}=0,244)$. Le Tableau 7 renseigne sur ces résultats.

Les résultats d'ajustement des données de la surface terrière $(\mathrm{G})$ et du diamètre $(\mathrm{D})$ de Lophira lanceolata aux différents modèles de régression permettent de noter que l'ajustement des données aux cinq modèles testés est significatif dans tous les cas au seuil de $5 \%$ puisque les probabilités sont égales à 0,001 . 
Tableau 1 : Statistique descriptive des paramètres dendrométriques des peuplements de Lophira lanceolata par formation végétale.

\begin{tabular}{|c|c|c|c|c|c|c|c|c|c|c|c|c|}
\hline \multicolumn{13}{|c|}{ Peuplement global } \\
\hline & \multicolumn{4}{|l|}{ FCSB } & \multicolumn{4}{|l|}{ SAB } & \multicolumn{4}{|l|}{ CJ } \\
\hline & D & $\mathbf{H}$ & $\mathbf{G}$ & $\mathbf{N}$ & D & $\mathbf{H}$ & $\mathbf{G}$ & $\mathbf{N}$ & D & $\mathbf{H}$ & $\mathbf{G}$ & $\mathbf{N}$ \\
\hline Placettes & 20,00 & 20,00 & 20,00 & 20,00 & 35,00 & 35,00 & 35,00 & 35,00 & 9,00 & 9,00 & 9,00 & 9,00 \\
\hline Moyenne & 22,17 & 10,35 & 0,52 & 266,11 & 18,06 & 8,27 & 0,36 & 210,47 & 21,48 & 8,20 & 0,50 & 90,12 \\
\hline Médiane & 21,50 & 10,23 & 0,49 & 250,00 & 16,63 & 7,88 & 0,27 & 200,00 & 20,33 & 7,95 & 0,44 & 77,78 \\
\hline Mode & 15,80 & 10,15 & 0,26 & 222,22 & 13,50 & 5,00 & 0,06 & 144,44 & 17,14 & 6,57 & 0,28 & 44,44 \\
\hline Variance & 22,68 & 3,10 & 0,07 & 7673,00 & 26,91 & 3,77 & 0,06 & 10435,31 & 15,75 & 2,40 & 0,04 & 3690,00 \\
\hline Minimum & 15,80 & 7,88 & 0,26 & 88,89 & 11,38 & 5,00 & 0,06 & 22,22 & 17,14 & 6,57 & 0,28 & 33,33 \\
\hline Maximum & 39,65 & 15,65 & 1,49 & 511,11 & 29,13 & 13,50 & 1,01 & 522,22 & 28,46 & 11,67 & 0,85 & 233,33 \\
\hline \multicolumn{13}{|c|}{ Lophira lanceolata } \\
\hline & \multicolumn{4}{|l|}{ FCSB } & \multicolumn{4}{|l|}{ SAB } & \multicolumn{4}{|l|}{ CJ } \\
\hline & D & $\mathbf{H}$ & $\mathbf{G}$ & $\mathbf{N}$ & D & $\mathbf{H}$ & $\mathbf{G}$ & $\mathbf{N}$ & D & $\mathbf{H}$ & $\mathbf{G}$ & $\mathbf{N}$ \\
\hline Placettes & 10,00 & 10,00 & 10,00 & 10,00 & 4,00 & 4,00 & 4,00 & 4,00 & 2,00 & 2,00 & 2,00 & 2,00 \\
\hline Moyenne & 25,50 & 15,21 & 0,63 & 18,89 & 18,13 & 9,08 & 0,43 & 19,44 & 19,84 & 8,12 & 0,41 & 11,11 \\
\hline Médiane & 24,40 & 13,13 & 0,55 & 16,67 & 16,03 & 7,98 & 0,30 & 16,67 & 19,84 & 8,12 & 0,41 & 11,11 \\
\hline Mode & 13,00 & 9,00 & 0,15 & 11,11 & 11,47 & 6,87 & 0,12 & 11,11 & 18,85 & 7,65 & 0,35 & 11,11 \\
\hline Variance & 71,37 & 37,15 & 0,13 & 83,68 & 60,27 & 8,99 & 0,17 & 113,17 & 1,94 & 0,43 & 0,01 & 0,00 \\
\hline Minimum & 13,00 & 9,00 & 0,15 & 11,11 & 11,47 & 6,87 & 0,12 & 11,11 & 18,85 & 7,65 & 0,35 & 11,11 \\
\hline Maximum & 36,00 & 26,50 & 1,13 & 33,33 & 29,00 & 13,50 & 1,01 & 33,33 & 20,82 & 8,58 & 0,47 & 11,11 \\
\hline
\end{tabular}

$F C S B=$ Forêt Claire et en Savane Boisée, $S A B=$ Savane Arborée et Arbustive, $(C J)$. Champ et jachère, D=Diamètre, $H=$ hauteur, $G=$ Surface terrière, $N=$ Densité d'arbre
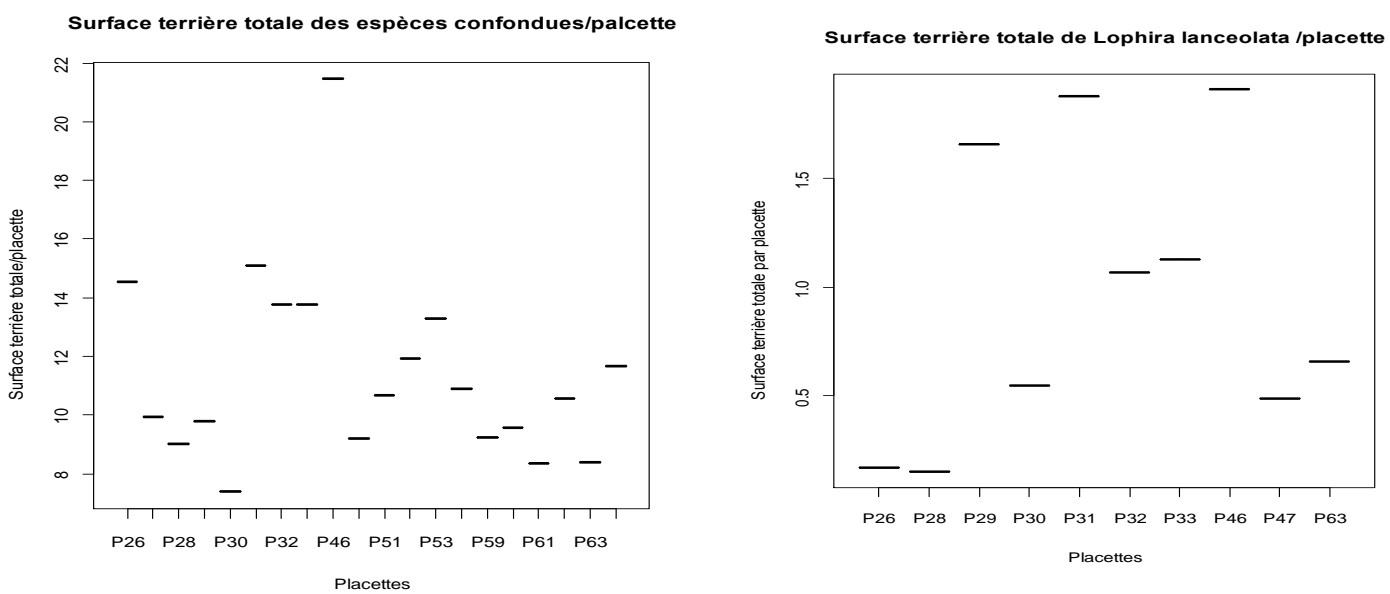

Figure 3 : Surface terrière totale de toutes les espèces confondues (a) et des arbres de Lophira lanceolata (b) au niveau de la forêt claire et savane boisée de Toui-Kilibo. 
Surface terrière totale de toutes les espèces condues/placett Surface terrière de Lophira lanceolata/placette
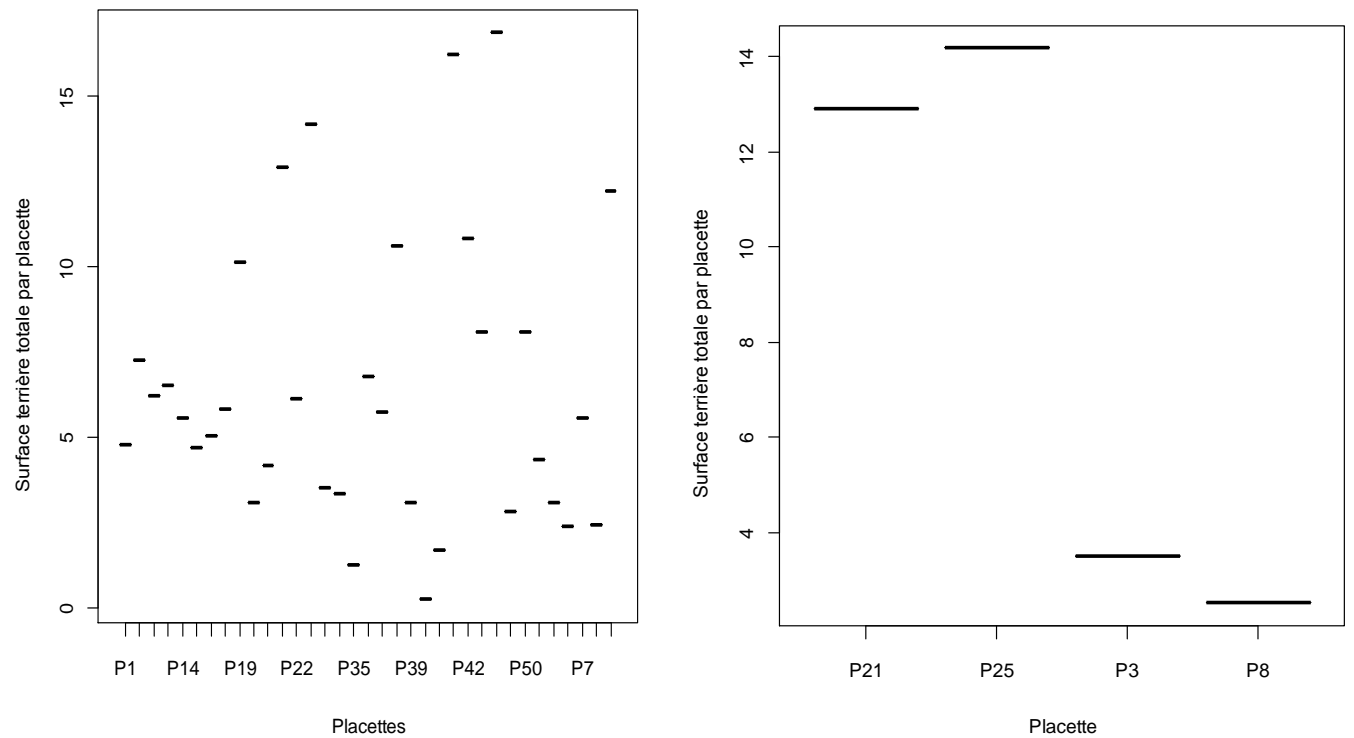

Figure 4 : Surface terrière totale de toutes les espèces confondues (a) et des arbres de Lophira lanceolata (b) au sein des placettes au niveau des savanes arborées et arbustives de la région de Ouèssè̀.

Surface terrière totale de toutes les espèces confondues/place
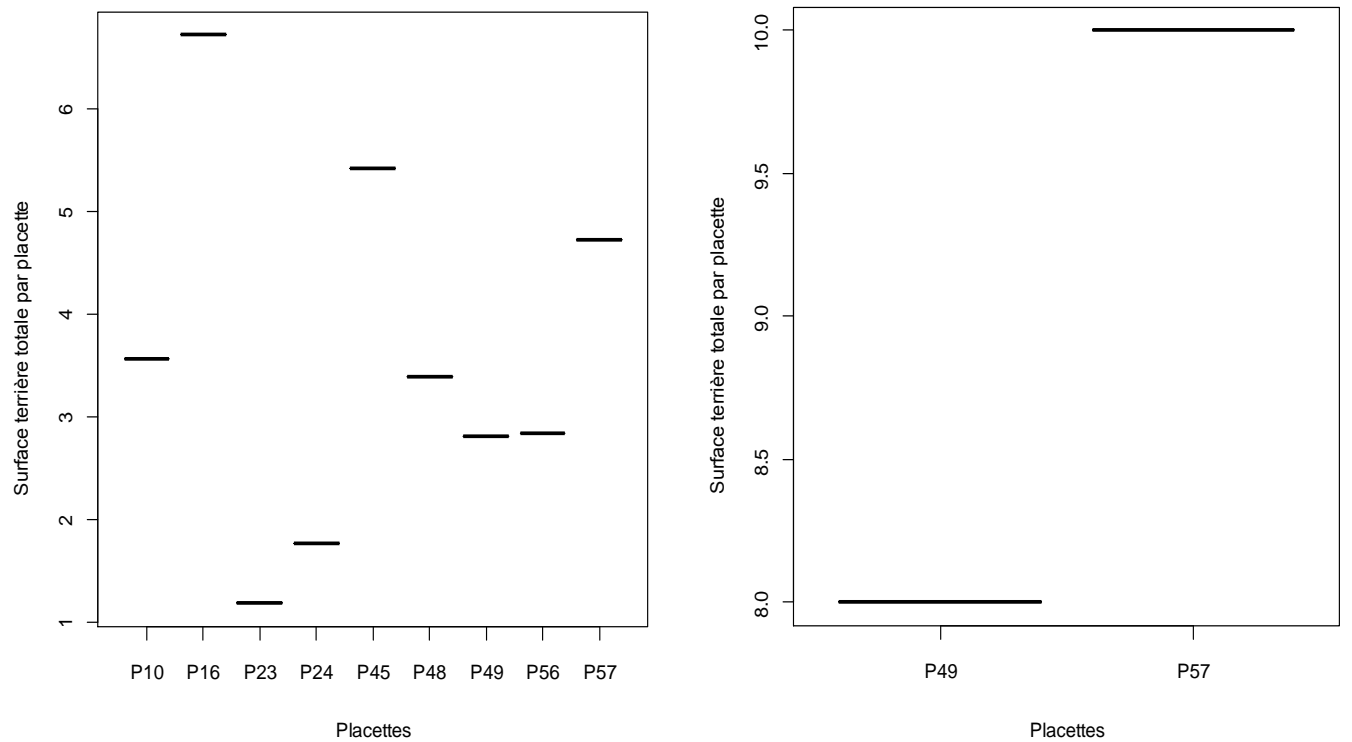

Figure 5 : Surface terrière totale de toutes les espèces confondues (a) et des arbres de Lophira lanceolata (b) au sein des placettes au niveau des champs et jachères de la région de Ouèssè. 


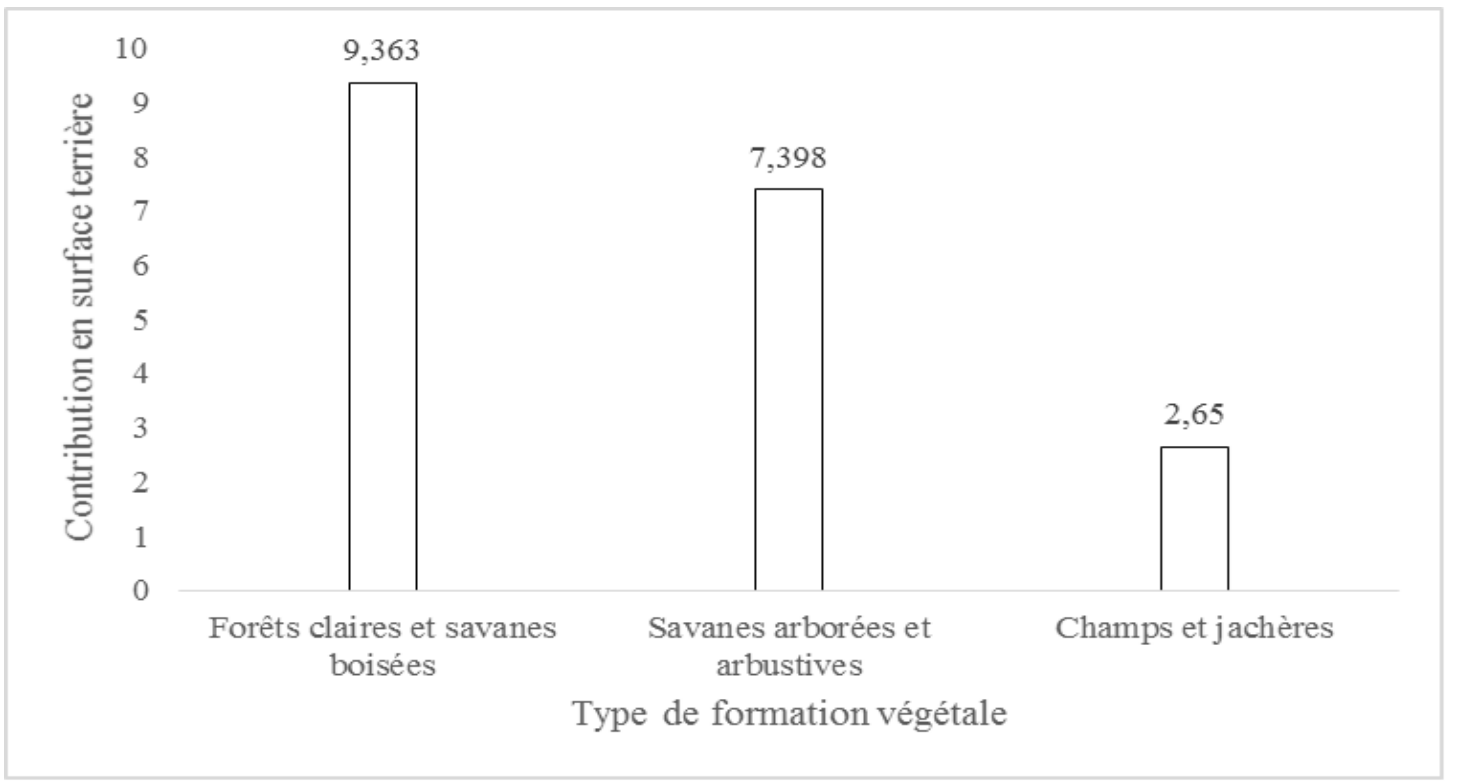

Figure 6 : Contribution en surface terrière des arbres de Lophira lanceolata.

Tableau 2 : Fréquence, densité relative, dominance relative, Amplitude d'habitat, et Indice de Blackman des arbres de Lophira lanceolata selon les formations végétales.

\begin{tabular}{llllll}
\hline & Fréquence & $\begin{array}{l}\text { Densité } \\
\text { relative }\end{array}$ & $\begin{array}{l}\text { Dominance } \\
\text { relative }\end{array}$ & $\begin{array}{l}\text { Amplitude } \\
\text { d'habitat }\end{array}$ & $\begin{array}{l}\text { Indice de } \\
\text { Blackman } \mathbf{I}_{\mathbf{B}}\end{array}$ \\
\hline Champs et jachères & 0,22 & 2,74 & 23,26 & 1,152 & 0 \\
\hline Savane arborée et arbustive & 0,11 & 1,06 & 14,70 & 1,071 & 5,821 \\
\hline Forêt claire et savane boisée & 0,50 & 3,55 & 4,23 & 1,186 & 4,43 \\
\hline
\end{tabular}

Tableau 3 : Paramètres dendrométriques des essences de valeur des peuplements à dominance Lophira lanceolata en champs et jachères.

\begin{tabular}{lrrrr}
\hline Espèces & $\begin{array}{l}\text { Densité, } \mathbf{N} \\
\text { (tiges/ha) }\end{array}$ & $\begin{array}{l}\text { Surface terrière, } \\
\mathbf{G}\left(\mathbf{m}^{2} / \mathbf{h a}\right)\end{array}$ & $\begin{array}{l}\text { Diamètre } \\
(\mathbf{c m})\end{array}$ & $\begin{array}{l}\text { Hauteur } \\
(\mathbf{m})\end{array}$ \\
\hline Afzelia africana & 5,56 & 0,68 & 13,00 & 5,80 \\
\hline Anogeissus leiocarpus & 5,56 & 0,63 & 12,50 & 8,00 \\
\hline Combretum glutinosum & 5,56 & 0,50 & 11,40 & 4,00 \\
\hline Ficus sur & 11,11 & 3,20 & 19,70 & 8,90 \\
\hline Khaya senegalensis & 5,56 & 3,11 & 28,10 & 12,50 \\
\hline Lannea barteri & 5,56 & 2,48 & 25,10 & 10,00 \\
\hline Lophira lanceolata & 11,11 & 0,41 & 19,84 & 8,12 \\
\hline Pterocarpus erinaceus & 22,22 & 6,80 & 20,08 & 8,08 \\
\hline Tectona grandis & 11,11 & 2,21 & 16,80 & 8,00 \\
\hline Vitellaria paradoxa & 11,11 & 12,24 & 38,50 & 10,80 \\
\hline Vitex doniana & 5,56 & 1,17 & 17,10 & 7,50 \\
\hline
\end{tabular}


Tableau 4 : Paramètres dendrométriques des essences de valeur des peuplements à dominance Lophira lanceolata en savanes arborée et arbustives.

\begin{tabular}{|c|c|c|c|c|}
\hline Espèces & $\begin{array}{l}\text { Densité, N } \\
\text { (tiges/ha) }\end{array}$ & $\begin{array}{l}\text { Surface terrière, } G \\
\left(\mathrm{~m}^{2} / \mathrm{ha}\right)\end{array}$ & $\begin{array}{l}\text { Diamètre } \\
(\mathrm{cm})\end{array}$ & $\begin{array}{l}\text { Hauteur } \\
(\mathrm{m})\end{array}$ \\
\hline Acacia sieberiana & 16,67 & 3,59 & 12,33 & 7,67 \\
\hline Annona senegalensis & 44,44 & 5,60 & 13,50 & 8,75 \\
\hline Anogeissus leiocarpus & 369,44 & 80,24 & 14,76 & 8,24 \\
\hline Burkea africana & 44,44 & 19,95 & 26,00 & 8,67 \\
\hline Clerodendron volubile & 33,33 & 2,01 & 11,50 & 6,00 \\
\hline Combretum collinum & 33,33 & 2,45 & 10,33 & 6,67 \\
\hline Crossopteryx febrifuga & 38,89 & 4,29 & 13,33 & 7,00 \\
\hline Daniella oliveri & 275,00 & 23,71 & 36,00 & 13,00 \\
\hline Ficus ovata & 5,56 & 22,23 & 54,00 & 13,00 \\
\hline Ficus sur & 33,33 & 1,49 & 10,00 & 6,50 \\
\hline Lannea acida & 16,67 & 39,11 & 50,00 & 11,50 \\
\hline Lannea barteri & 52,78 & 13,13 & 21,67 & 8,33 \\
\hline Lonchocarpus sericeus & 22,22 & 8,23 & 18,67 & 11,00 \\
\hline Lophira lanceolata & 19,44 & 0,43 & 18,13 & 9,08 \\
\hline Piliostigma tonningii & 83,33 & 4,64 & 17,00 & 6,50 \\
\hline Prosopis africana & 25,00 & 17,76 & 33,50 & 10,50 \\
\hline Pteleopsis suberosa & 5,56 & 2,19 & 12,00 & 6,00 \\
\hline Sarcocephalus latifolius & 8,33 & 0,79 & 10,00 & 5,00 \\
\hline Terminalia laxiflora & 66,67 & 4,20 & 13,33 & 8,33 \\
\hline Vitellaria paradoxa & 191,67 & 41,65 & 20,09 & 7,91 \\
\hline Vitex doniana & 8,33 & 48,83 & 80,00 & 40,00 \\
\hline
\end{tabular}

Tableau 5 : Paramètres dendrométriques des essences de valeur des peuplements à dominance Lophira lanceolata en Forêt claire et Savane boisée.

\begin{tabular}{|c|c|c|c|c|}
\hline Espèces FCSB en groupement & $\begin{array}{l}\text { Densité, N } \\
\text { (tiges/ha) }\end{array}$ & $\begin{array}{l}\text { Surface terrière, } \\
\text { G }\left(\mathbf{m}^{2} / \mathbf{h a}\right)\end{array}$ & Diamètre & Hauteur \\
\hline Anogeissus leiocarpus & 74,44 & 34,48 & 19,89 & 12,25 \\
\hline Burkea africana & 33,33 & 16,90 & 27,33 & 12,05 \\
\hline Combretum collinum & 27,78 & 9,24 & 15,60 & 7,15 \\
\hline Combretum glutinosum & 10,00 & 3,26 & 14,80 & 7,25 \\
\hline Combretum molle & 1,11 & 0,20 & 10,60 & 7,50 \\
\hline Combretum sp & 3,33 & 0,78 & 14,95 & 7,25 \\
\hline Crossopteryx febrifuga & 26,67 & 6,90 & 15,77 & 7,03 \\
\hline
\end{tabular}




\begin{tabular}{lcccr}
\hline Daniella oliveri & 57,78 & 41,96 & 27,80 & 12,29 \\
\hline Detarium microcarpum & 7,78 & 1,90 & 16,63 & 8,50 \\
\hline Hymenocardia acida & 17,78 & 6,66 & 15,63 & 7,79 \\
\hline Isoberlinia doka & 46,67 & 47,86 & 25,00 & 13,84 \\
\hline Lannea acida & 8,89 & 1,72 & 21,05 & 11,50 \\
\hline Lannea barteri & 11,11 & 5,36 & 22,00 & 8,17 \\
\hline Lophira lanceolata & 18,89 & 0,63 & 25,50 & 15,21 \\
\hline Monotes kerstingii & 18,89 & 0,92 & 23,00 & 7,00 \\
\hline Parinari curatellifolia & 16,67 & 9,32 & 21,93 & 6,77 \\
\hline Pericopsis laxiflora & 8,89 & 3,90 & 18,57 & 9,67 \\
\hline Piliostigma toningii & 26,67 & 3,62 & 12,90 & 6,08 \\
\hline Prosopis africana & 6,67 & 2,44 & 26,45 & 15,00 \\
\hline Sarcocephalus latifolius & 1,11 & 0,44 & 16,00 & 5,00 \\
\hline Terminalia avicennioides & 12,22 & 0,60 & 10,73 & 6,37 \\
\hline Terminalia laxiflora & 6,67 & 2,32 & 14,67 & 8,83 \\
\hline Vitellaria paradoxa & 25,56 & 17,08 & 21,04 & 8,61 \\
\hline Xeroderris stuhlmannii & 8,89 & 1,68 & 15,40 & 8,50
\end{tabular}

Tableau 6 : Régression linéaire entre la surface terrière $(\mathrm{G})$, le diamètre $(\mathrm{D})$ et la hauteur $(\mathrm{H})$ des arbres de Lophira lanceolata.

Lophira lanceolata de la Forêt claire et savane boisée

\begin{tabular}{|c|c|c|c|c|c|c|}
\hline & Constants & $\mathrm{t}$ & $\mathrm{P}$ & $\mathbf{R}^{2}$ ajusté & Fisher & Sig \\
\hline (Constant) & $-0,492$ & $-10,924$ & 0,001 & $98 \%$ & 383,335 & 0,001 \\
\hline Diamètre & 0,043 & 22,465 & 0,001 & & & \\
\hline Hauteur & 0,001 & 0,596 & 0,561 & & & \\
\hline
\end{tabular}

Equation du modèle : $\mathrm{G}=-0,492+0,043 \mathrm{D} * *+0,001 \mathrm{H}$

Lophira lanceolata de la savane arborée et boisée

\begin{tabular}{|c|c|c|c|c|c|c|}
\hline SAB & Constants & $\mathbf{t}$ & $\mathbf{P}$ & $\mathbf{R}^{2}$ ajusté & Fisher & Sig \\
\hline (Constant) & $-0,164$ & $-0,637$ & 0,558 & $96,70 \%$ & 89,826 & 0,001 \\
\hline Diamètre & 0,044 & 9,45 & 0,001 & & & \\
\hline Hauteur & 0,033 & $-0,9$ & 0,419 & & & \\
\hline
\end{tabular}

Equation du modèle : $\mathrm{G}=-0,164+0,044 \mathrm{D} * *+0,033 \mathrm{H}$ 
Tableau 7: Ajustement de la hauteur et du diamètre.

\begin{tabular}{llll}
\hline Modèles & Equations & Probabilités & $\mathbf{R}^{\mathbf{2}}$ \\
\hline 1 - Linéaire simple & $\mathrm{H}=4,315+43,123 \mathrm{D}^{*}$ & 0,018 & 0,276 \\
2 - Trorey & $\mathrm{H}=12,289-28,732 \mathrm{D}$ & 0,244 & 0,5 \\
& $+143,336 \mathrm{D}^{2}$ & & \\
$\mathbf{3}$ - Hendricksen & $\mathrm{H}=28,959+9,528 \mathrm{LnD}^{*}$ & 0,024 & 0,247 \\
$\mathbf{4}$ - Stoffels et de Van Soest & $\mathrm{LnH}=3,500+0,595 \mathrm{LnD}^{*}$ & 0,026 & 0,243 \\
$\mathbf{5}$ - Avery et Burkart & $\mathrm{LnH}=3,178-0,1171 / \mathrm{D}^{*}$ & 0,038 & 0,257 \\
\hline
\end{tabular}

Tableau 8 : Ajustement de la surface terrière et du diamètre.

\begin{tabular}{llll}
\hline Modèles & Equations & P & $\mathbf{R}^{\mathbf{2}}$ \\
\hline 1 - Linéaire simple & $\mathrm{G}=-0,485+4,372 \mathrm{D}^{* *}$ & 0,001 & 0,98 \\
$\mathbf{2}$ - Trorey & $\mathrm{G}=0,027 \times 10^{-10}-4,39 \times 10^{-9} \mathrm{D}-0,722 \mathrm{D}^{2^{* *}}$ & 0,001 & 1 \\
$\mathbf{3}$ - Hendricksen & $\mathrm{G}=2,032+0,979 \mathrm{LnD} \mathrm{D}^{* *}$ & 0,001 & 0,92 \\
$\mathbf{4}$ - Stoffels et de Van Soest & $\mathrm{LnG}=2,166+2,00 \mathrm{LnD}$ & 0,001 & 1 \\
$\mathbf{5}$ - Avery et Burkart & $\mathrm{LnG}=1,174-0,415(1 / \mathrm{D})^{* *}$ & 0,001 & 0,98 \\
\hline
\end{tabular}

\section{DISCUSSION}

L'étude dendrométrique des arbres de Lophira lanceolata est faite de la forêt classée de Toui-Kilibo et ses environs au Bénin. La caractérisation dendrométrique est un élément essentiel pour la réalisation de plans de gestion et de conservation durables Fandohan et al. (2008). Les paramètres dendrométriques de l'espèce varient en fonction des types de formation végétale. En considérant les pieds de Lophira lanceolata ayant un $\mathrm{dbh} \geq 10 \mathrm{~cm}$, l'espèce est plus dense à l'intérieur de la forêt et de la savane qu'en jachère. La densité de Lophira lanceolata est de 19,44 arbres/ha au niveau de la savane arborée et arbustive, alors qu'elle est de 11,11 arbres/ha au niveau des champs et jachères. Les valeurs de ces densités sont inférieures à celles trouvées par Dicko et al. (2018) dans le district phytogéographique de Bassila au Bénin. Ces auteurs ont trouvé des densités de Lophira lanceolata allant jusqu'à $91 \mathrm{arbres} / \mathrm{ha}$. Mais ceci se justifie par le fait que ces auteurs ont pris en compte des arbres de Lophira lanceolata supérieur ou égal à 5 centimètres de diamètre. Alors que notre étude a fait cas des arbres dont le diamètre est supérieur ou égal à 10 centimètres. Les valeurs des densités trouvées dans notre étude sont supérieures à celles trouvées par Garba et al. (2020) sur les individus de Tamarindus indica dans trois communes de Niger. Ces auteurs ont obtenu des valeurs des densités de Tamarindus indica comprises entre 1 à 10 individus/ha.

Le diamètre et la hauteur de l'espèce sont plus élevés à l'intérieur de la forêt que dans les périphéries. Le diamètre moyen de l'espèce qui est de $25,50 \mathrm{~cm}$ au niveau de la forêt alors qu'il est de $18,13 \mathrm{~cm}$ au niveau de la savane. Ces valeurs sont inférieures à celles trouvés par Fachola et al. (2019) sur les 
diamètres moyens de Daniella oliveri qui sont de $36,93 \mathrm{~cm}$ et $26,93 \mathrm{~cm}$ respectivement pour le district phytogéographique de Pobè et de Plateau au Bénin. Ces faibles valeurs de diamètre sont relatives aux menaces anthropiques causées par les agriculteurs pour diverses activités. Pour Moumouni et al. (2017), cela pourrait s'expliquer par les défrichements culturaux au cours desquels on assiste à l'abattage systématique des gros arbres sur de très grande superficie.

La contribution en surface terrière de Lophira lanceolata décroît quand on passe de la forêt claire et savane boisée, de la savane arborée et arbustive, vers les champs et jachères (donc de la formation naturelle vers les formations dégradées).

La densité de Lophira lanceolata trouvée en forêt claire et savane boisée $(18,89$ arbres/ha) est semblable à celle de Monotes kerstingii. Mais cette valeur de densité est supérieure aux densités de Hymenocardia acida (17,78), Parinari curatellifolia $(16,67)$, Terminalia avicennioides (12,22), Lannea barteri (11,11), Combretum glutinosum (10), Lannea acida (8,89), Pericopsis laxiflora $(8,89)$, Xeroderris stuhlmannii $(8,89)$, Detarium microcarpum (7,78), Prosopis africana $(6,67)$, Terminalia laxiflora $(6,67)$, Combretum sp (3,33), Combretum molle $(1,11)$, Sarcocephalus latifolius $(1,11)$.

Mais les espèces comme Anogeissus leiocarpus $(74,44)$, Daniella oliveri $(57,78)$, Isoberlinia doka (46,67), Burkea africana (33,33), Combretum collinum (27,78), Crossopteryx febrifuga (26,67), Piliostigma toningii $(26,67)$, Vitellaria paradoxa $(25,56)$ ont une densité élevée que la densité de Lophira lanceolata à l'intérieure de la forêt. Ces espèces sont d'une grande utilité, elles sont protégées pour en faire des bois d'œuvre.

A l'intérieur de la forêt, le diamètre de Lophira lanceolata $(22,68 \mathrm{~cm})$ est supérieur au diamètre de Vitellaria paradoxa (17,08), Burkea africana (16,9), Parinari curatellifolia (9,32), Combretum collinum $(9,24)$,
Crossopteryx febrifuga (6,9), Hymenocardia acida (6,66), Lannea barteri (5,36), Pericopsis laxiflora (3,9), Piliostigma toningii $(3,62)$, Combretum glutinosum $(3,26)$, Prosopis africana $(2,44)$, Terminalia laxiflora $(2,32)$, Detarium microcarpum (1,9), Lannea acida $(1,72)$, Xeroderris stuhlmannii $(1,68)$, Monotes kerstingii (0,92), Combretum sp (0,78), Terminalia avicennioides (0,6), Sarcocephalus latifolius $(0,44)$, Combretum molle $(0,2)$. Cela confirme que l'espèce a trouvé des conditions nécessaires qui ont permis d'accroitre son diamètre et cette espèce a été conservée. En effet, Biwolé et al. (2012) avaient signalé que le diamètre maximal de Lophira lanceolata peut atteindre $70 \mathrm{~cm}$. Cette valeur est supérieure à celle trouvée dans notre étude.

Mais les espèces comme Isoberlinia doka (47,86), Daniella oliveri (41,96), Anogeissus leiocarpus $(34,48)$ ont des diamètres supérieurs à celui de Lophira lanceolata. Ces espèces sont des espèces de bois d'œuvre qui sont souvent protégés pour leur utilité grandiose.

De plus, la hauteur en mètre de Lophira lanceolata trouvée à l'intérieur de la forêt est supérieure à celle de Prosopis africana (15), Isoberlinia doka $(13,84)$, Daniella oliveri (12,29), Anogeissus leiocarpus (12,25), Burkea africana (12,05), Lannea acida (11,5), Pericopsis laxiflora $(9,67), \quad$ Terminalia laxiflora $(8,83)$, Vitellaria paradoxa $(8,61)$, Detarium microcarpum (8,5), Xeroderris stuhlmannii $(8,5)$, Lannea barteri $(8,17)$, Hymenocardia acida $(7,79)$, Combretum molle $(7,5)$. Ces résultats confirment que si Lophira lanceolata n'est pas menacée par les actions anthropiques, elle peut avoir des paramètres dendrométriques élevés.

La dominance relative la plus élevée de Lophira lanceolata 23,26\% retrouvée au niveau des champs et jachères s'apparente à celle de Scorodophloeus zenkeri (24\%) dans le bloc nord du dispositif permanent de Yoko au RD Congo (Basele liyele, 2013). 
Les sensibilisations doivent être faites à l'endroit des paysans pour conserver Lophira lanceolata dans leurs champs. Nous recommandons aux paysans d'épargner le plus possible l'espèce au moment des défrichements de leurs espaces culturaux. L'introduction de cette espèce dans les champs sera nécessaire pour sa meilleure conservation.

\section{Conclusion}

Cette recherche a porté sur la connaissance des paramètres dendrométriques de Lophira lanceolata de la forêt classée de Toui-Kilibo et ses environs au Bénin. Lophira lanceolata contribue à $9,363 \%$ en surface terrière en forêt claire et savane boisée de TouiKilibo, 7,398\% en savane arborée et arbustive et $2,65 \%$ en champs et jachères. Au niveau des champs et jachères, 10 espèces ayant un diamètre supérieur à $10 \mathrm{~cm}$ compagnes avec Lophira lanceolata. En savanes arborée et arbustives, 20 espèces compagnes l'espèce sont recensées. En Forêt claire et Savane boisée, 23 espèces ligneuses qui compagnes l'espèce sont recensées. La connaissance des paramètres dendrométriques de Lophira lanceolata dans cette zone d'étude permet d'avoir une base de données fiable de cette espèce d'utilités multiples. Une meilleure protection de Lophira lanceolata engendre des individus de gros diamètres qui sont utiles pour le bois d'œuvre et de service. Ce qui augmente la surface terrière. La politique de domestication de Lophira lanceolata est nécessaire pour accroître la densité de cette espèce dans le secteur d'étude.

\section{CONFLIT D'INTERETS}

Les auteurs déclarent qu'ils n'ont aucun conflit d'intérêt concernant ce travail.

\section{CONTRIBUTIONS DES AUTEURS}

RWA a élaboré le protocole de travail et a fait la collecte des données. TOL et GJD ont validé la méthodologie, ont vérifié la crédibilité des données collectées, ont appuyé l'analyse des données, ont supervisé le document.

\section{REMERCIEMENTS}

Au terme de ce travail, nous remercions vivement le Laboratoire d'Ecologie Appliquée (LEA) de l'Université d'Abomey-Calavi pour nous avoir donné un local approprié pour la réflexion scientifique. Nous remercions également les personnes qui nous ont aidés sur le terrain pour la bonne marche de la collecte des données.

\section{REFERENCES}

Basele Liyele E. 2013. Contribution à l'étude floristique et dendrométrique de deux peuplements à Prioriaoxyphylla J. Léonard. dans le bloc nord du dispositif permanent de Yoko (Ubundu, Province Orientale, RD Congo). Travail de fin de cycle Présenté en vue de l'obtention du grade de gradué en Sciences. Département d'Ecologie et Gestion des Ressources Végétales, Faculté des Sciences, Université de Kisangani, 35 p.

Biwolé AB, Bourland N, Daïnou K, Doucet JL. 2012. Définition du profil écologique de l'azobé, Lophira alata, une espèce ligneuse africaine de grande importance : synthèse bibliographique et perspectives pour des recherches futures. Biotechnol. Agron. Soc Environ., 16(2) : 217-228.

Dicko A, Natta AK, Biaou HSS, Balagueman R, Gouwakinnou G. 2017. Typology and structural characterization of Lophira lanceolata populations in Benin (west africa). Rev. CAMES, 5(1) : 36-43.

Dicko A, Natta AK, Gouwakinnou G, MOUTOUAMA JK. 2018. Prédiction spatiale de la distribution de Lophira lanceolata Tiegh. Ex Keay au Bénin: Changement climatiques et habitats favorables. In thèse de Doctorat, Université de Parakou, 113-129.

Djogbenou CP, Arouna O, Glèlè Kakaï RL, Sinsin BA. 2008. Critères et indicateurs 
de participation des populations locales à l'aménagement forestier au Bénin. Bulletin de la Recherche Agronomique du Bénin, 62 : 1-13.

Fachola BO, Gbèsso GHF, Lougbégnon OT, Agossou N. 2019. Paramètres dendrométriques et structuraux de Parkia biglobosa (Jacq.) R. Br. et de Daniellia oliveri (Rolfe) Hutch. \& Dalziel dans les phytodistricts Pobè et Plateau au Bénin. International Journal of Biological and Chemical Sciences, 13(2): 652-661. DOI: https://dx.doi.org/10.4314/ijbcs.v13i2.7

Fandohan B, Glèlè Kakaï R, Sinsin B, Pelz D. 2008. Caractérisation dendrométrique et spatiale de trois essences ligneuses médicinales de la forêt classée de WariMaro au Bénin. Rev. Ivoir. Sci. Technol., 12: 173-186.

Fonton HN, Sagbo AAP. 2004. Modélisation des distributions des paramètres dendrométriques d'une espèce ligneuse dominante en peuplement naturel. Ann. For. Sci., 61: 545-549. DOI: 10.1051/forest:2004049

Fortin M, Bernier S, Saucier JP, Labbé F. 2009. Une relation hauteur-diamètre tenant compte de l'influence de la strate et du climat pour 20 espèces commerciales du Québec. Mémoire de recherche en foresterie 153, 23 pages.

Garba A, Amani A, Douma S, Sina AKS, Mahamane A. 2020. Structure des populations de Tamarindus indica $\mathrm{L}$. dans la zone Sud-Ouest du Niger. International
Journal of Biological and Chemical Sciences, $\quad$ 14(1): 126-142. DOI: https://dx.doi.org/10.4314/ijbcs.v14i1.11 Igué AM, Houndagba CJ, Worou R, Gaiser T, Mensah GA, Stahr K. 2010. Aspects de la dynamique de l'occupation du sol et du projet d'aménagement participatif de la forêt classée de Toui-Kilibo au Bénin. Rev.Sc. Env? Univ., Lomé (Togo), $\mathrm{n}^{\circ} 006$.

Moumouni YI, Arouna O, Zakari S. 2017. Diversité floristique et structure des formations végétales dans le district phytogéographique du Borgou-nord au Bénin (secteur de l'arrondissement de Bagou). Notes Scientifiques, Homme et Société, Faculté des Sciences de l'Homme et de la Société, Université de Lomé, 7 : 63-80.

Sagbo AA. 2000. Etude des Caractéristiques Dendrométriques des Peuplements Naturels à Dominance Isoberlinia spp : Cas de la Forêt Classée de l'Ouémé supérieur au Nord du Bénin. Thèse Pour l'obtention du Diplôme d'Ingénieur Agronome, Université Nationale du Bénin, $93 \mathrm{p}$.

Thiombiano A, Glèlè Kakaï R, Bayen $\mathrm{P}$, Boussim JI, Mahamane A. 2016. Méthodes et dispositifs d'inventaires forestiers en Afrique de l'Ouest : Etat des lieux et propositions pour une harmonisation. Annales des Sciences Agronomiques, 20: 15-31. 